\title{
HEAT KERNEL ON CONNECTED SUMS OF RIEMANNIAN MANIFOLDS
}

\author{
Alexander Grigor'yan And Laurent SAloff-Coste
}

\section{Introduction}

This note is about the heat kernel on a connected sum $M$ of non-compact manifolds $M_{1}, M_{2}, \ldots, M_{k}$ assuming that one knows enough about the heat kernels for each $M_{i}$ individually (which is the case when $M_{i}$ are complete manifolds of non-negative Ricci curvature). We announce here matching uniform upper and lower bounds for the heat kernel on such manifolds $M$. The proofs will be given elsewhere.

For an arbitrary Riemannian manifold $M$, denote by $\Delta$ the Laplace operator of the Riemannian metric of $M$ and by $p(t, x, y)$ the heat kernel, that is, the smallest positive fundamental solution to the heat equation $u_{t}=\Delta u$ on $\mathbb{R}_{+} \times M$ (here $x, y \in M$ and $t>0$ ). Alternatively, $p(t, x, y)$ can be defined as the kernel of the semigroup $\exp (t \Delta)$. Yet another definition of $p(t, x, y)$ is that it is the density of the transition probability of the Brownian motion on $M$ generated by the operator $\Delta$.

In $\mathbb{R}^{n}$, the heat kernel is given by the classical formula

$$
p(t, x, y)=\frac{1}{(4 \pi t)^{n / 2}} \exp \left(-\frac{|x-y|^{2}}{4 t}\right) .
$$

On a general manifold $M$, one may hope to obtain some estimates of the heat kernel related to the geometry of $M$. In the past two decades, this question has received considerable attention from many authors. This was partially motivated by various important applications of heat kernel bounds although the question of understanding the interplay between the heat kernel and the geometry is interesting by itself. The celebrated theorem of Li and Yau [16] says that if $M$ is a geodesically complete manifold with non-negative Ricci curvature then

$$
\frac{c}{V(x, \sqrt{t})} \exp \left(-\frac{d^{2}(x, y)}{c t}\right) \leq p(t, x, y) \leq \frac{C}{V(x, \sqrt{t})} \exp \left(-\frac{d^{2}(x, y)}{C t}\right)
$$

Here $V(x, r)$ is the Riemannian volume of the geodesic ball $B(x, r)$ of radius $r$ centered at $x \in M ; d(x, y)$ is the geodesic distance between the points $x, y \in M$;

\footnotetext{
Received March 18, 1999.

Alexander Grigor'yan's research supported by EPSRC Fellowship B/94/AF/1782 (Eng-

Laurent Saloff-Coste's research supported by NSF grant DMS 9802855.
} land). 
$C, c$ are positive constants. There are many other settings when certain heat kernel bounds are known — see for example [1], [3], [4], [7], [8], [11], [12], [18], [17].

In most known estimates the heat kernel is controled by two multiplicative terms: a Gaussian factor $\exp \left(-\frac{d^{2}(x, y)}{C t}\right)$ and a long time decay term which is some function of time $t$ as in (1) and (2). The reason is that in most situations considered previously the manifold $M$ possesses a certain degree of homogeneity so that $p(t, x, y)$ depends on $x$ and $y$ mainly via the distance $d(x, y)$. At the present time, there are no methods yielding satisfactory heat kernel bounds for highly inhomogeneous manifolds having substantially different geometric properties in different parts. The manifolds with ends considered in this note can be regarded as the simplest case of such non-homogeneous manifolds.

The main results are Theorems 1 and 4 . Since the results are not simple, we first explain them for connected sums of Euclidean spaces (Section 2). The general case is considered in Sections 3 and 4.

\section{Connected sums of Euclidean spaces}

Let $M_{1}, M_{2}, \ldots, M_{k}$ be a finite family of geodesically complete non-compact Riemannian manifolds. We say that a manifold $M$ is a connected sum of $M_{i}$ 's and write

$$
M=M_{1} \# M_{2} \# \cdots \# M_{k}
$$

if, for some compact $K \subset M$ (which will be called the central part of $M$ ), the exterior $M \backslash K$ is a disjoint union of open sets $E_{1}, E_{2}, \ldots$, such that each $E_{i}$ is isometric to $M_{i} \backslash K_{i}$, for some compact $K_{i} \subset M_{i}$ (in fact, we will identify $E_{i}$ and $\left.M_{i} \backslash K_{i}\right)$. In other words, in order to obtain $M$, one cuts out holes $K_{i}$ in each $M_{i}$ and glues together the rest of $M_{i}$, that is $E_{i}$, over some compact $K$. Of course, a connected sum is not uniquely defined. When writing $M_{1} \# M_{2} \# \cdots$, we refer to any manifold which can be obtained in this way.

Another point of view is to regard $M$ as a manifold with ends $E_{i}$ assuming that each end $E_{i}$ can be closed to a complete manifold $M_{i}$. Sometimes it is convenient to say that $M_{i}$ 's are the ends of $M$.

The connected sum $\mathbb{R}^{n} \# \mathbb{R}^{n}$ was considered by Kuz'menko and Molchanov [15] as early as in 1979 as an example of a manifold where the Liouville property for bounded harmonic functions fails. However, even for this simple manifold, no uniform bounds of the heat kernel was known, except for partial estimates [2], [19]. Our main results - Theorems 1 and 4, provide sharp uniform heat kernel estimates for a rather wide class of connected sums $M_{1} \# M_{2} \# \cdots \# M_{k}$. In particular, they cover (but are not restricted to) the case when $M_{i}$ are manifolds with non-negative Ricci curvature.

In this section, we will explain our results in the situation when each $M_{i}$ is similar, at infinity, to an Euclidean space. To be precise, let us fix some (large) integer $N$ and define the manifold $\mathcal{R}^{n}:=\mathbb{R}^{n} \times \mathbb{S}^{N-n}$, for all integers $n$ from 1 to $N$. Clearly, $\mathcal{R}^{n}$ has topological dimension $N$ whereas, on a large scale, it 
resembles $\mathbb{R}^{n}$. It is natural to say that the asymptotic dimension of $\mathcal{R}^{n}$ is $n$. It makes sense to consider the connected sums of $\mathcal{R}^{n}$ 's with different $n$ since all those manifolds have the same topological dimension $N$. Hence, the main example of this section is the manifold $M=\mathcal{R}^{n_{1}} \# \mathcal{R}^{n_{2}} \# \cdots \# \mathcal{R}^{n_{k}}$.

E.B. Davies [9] considered certain one-dimensional model spaces with ends having asymptotic dimensions $n_{1}, n_{2}, \ldots$ and observed some interesting phenomena for the heat kernel long time behavior. For example, he showed that, if all $n_{i} \neq 2$ and some $n_{i}>2$ then, for a fixed point $x$ and for $t \rightarrow \infty$,

$$
p(t, x, x) \leq C t^{-n / 2}
$$

where

$$
n=\min _{i}\left\{2+\left|n_{i}-2\right|\right\} .
$$

In particular, the contributions to $n$ of the ends with the asymptotic dimensions 1 and 3 are equal. Indeed, if $n_{i}=1$ or 3 then $2+\left|n_{i}-2\right|=3$. E.B. Davies conjectured that the same is true for connected sums of manifolds, and the positive answer to his conjecture follows from our results (see Corollary 5). Moreover, one can see that there is a certain duality between the manifolds of asymptotic dimensions less than 2 and greater than 2 .

For the manifold $M=\mathcal{R}^{n}$, the following estimate of the heat kernel is wellknown:

$$
p(t, x, y) \asymp \frac{1}{\min \left(t^{N / 2}, t^{n / 2}\right)} \exp \left(-\frac{d^{2}(x, y)}{c t}\right),
$$

$d(x, y)$ being the geodesic distance on $M$. Here the sign $\asymp$ means that the ratio of the right-hand side and of the left-hand side is bounded from above and from below by some positive constants, for all values of $x, y \in M$ and $t>0$. We always follow the convention that the constant $c$ in the Gaussian exponential term may be different for the upper and for the lower bounds.

Consider now the manifold $M=\mathcal{R}^{n} \# \mathcal{R}^{m}$ where both $n, m>2$. If $t \leq 1$ then the estimate (3) holds again. Assume in the sequel $t>1$. For any point $x \in M$, denote

$$
|x|:=\sup _{z \in K} d(x, z) .
$$

The point $x \in M$ lies either in the central part $K$ or in one of two ends $\mathcal{R}^{n} \backslash K$, $\mathcal{R}^{m} \backslash K$. Let $x \in \mathcal{R}^{n} \backslash K$ and $y \in \mathcal{R}^{m} \backslash K$. Then we claim that

$$
p(t, x, y) \asymp\left(\frac{1}{t^{m / 2}|x|^{n-2}}+\frac{1}{t^{n / 2}|y|^{m-2}}\right) \exp \left(-\frac{d^{2}(x, y)}{c t}\right),
$$

which is a special case of Theorem 1 below. However, even (4) exhibits some new phenomena. Let us consider two asymptotic regimes. 
(a) Long time regime when $t \rightarrow \infty$ whereas $|x|$ and $|y|$ stay bounded. Clearly, (4) implies

$$
p(t, x, y) \asymp \frac{1}{t^{\min (n, m) / 2}}
$$

so that the long time behaviour of the heat kernel is determined by the end with the smallest asymptotic dimension.

(b) Medium time regime when $t \rightarrow \infty$ and $|x| \asymp|y| \asymp \sqrt{t}$. Then (4) implies

$$
p(t, x, y) \asymp \frac{1}{t^{n / 2+m / 2-1}} .
$$

In particular, for the manifold $M=\mathbb{R}^{n} \# \mathbb{R}^{n}$, in the medium time regime, we have

$$
p(t, x, y) \asymp \frac{1}{t^{n-1}} .
$$

Note that even this estimate is new (see [2] for an upper bound and [19] for a lower bound in the medium time regime). For comparison, if $x$ and $y$ stay in the same end and $|x| \asymp|y| \asymp \sqrt{t}$ then $p(t, x, y) \asymp t^{-n / 2}$ as for the heat kernel of $\mathbb{R}^{n}$ (see (8) below). Since $n-1>n / 2$, the estimate (6) reflects a bottleneck effect: for $|x| \asymp|y| \asymp \sqrt{t}$, the heat kernel $p(t, x, y)$ is substantially smaller if $x, y$ are in different ends than if they are in the same end.

Each term in (4) has a clear probabilistic meaning. Let $X_{t}$ be the Brownian motion on $M$, that is, the diffusion generated by the Laplace operator $\Delta$. Then $p(t, x, y)$ is the density of the probability that $X_{t}$ hits $y$ at time $t$ after starting at $x$. Roughly speaking, there are two possibilities for a continuous path to get from $x$ to $y$ : either $X_{t}$ spends most time in $\mathcal{R}^{m}$ or in $\mathcal{R}^{n}$. The probability of the former is of the order $\frac{1}{t^{m / 2}|x|^{n-2}}$ and of the latter is of the order $\frac{1}{t^{n / 2}|y|^{m-2}}$.

Another new effect can be observed when considering connected sums of at least three manifolds. Let

$$
M=\mathcal{R}^{n_{1}} \# \mathcal{R}^{n_{2}} \# \cdots \# \mathcal{R}^{n_{k}}
$$

where all $n_{i}>2$. Denote

$$
n=\min _{i} n_{i}
$$

Assuming as above that $t>1, x \in \mathcal{R}^{n_{i}} \backslash K$ and $y \in \mathcal{R}^{n_{j}} \backslash K$ where $i \neq j$, we have the following estimate

$$
p(t, x, y) \asymp\left(\frac{1}{t^{n_{j} / 2}|x|^{n_{i}-2}}+\frac{1}{t^{n_{i} / 2}|y|^{n_{j}-2}}+\frac{1}{t^{n / 2}|x|^{n_{i}-2}|y|^{n_{j}-2}}\right) e^{-\frac{d^{2}(x, y)}{c t}} .
$$

The first two terms here have the same interpretation as above. The third term is dominated by one of the first two if $n=n_{i}$ or $n=n_{j}$ (which is the case, in particular, when $k=2$ ). However, if $n<n_{i}$ and $n<n_{j}$ (which may happen only if $k \geq 3$ ) then the third term dominates, for $t$ large enough. It roughly 
corresponds to the probability that $X_{t}$ goes from $x$ to $y$ spending a long time in the end with the smallest asymptotic dimension $n$. Hence, the most probable way of getting from $x$ to $y$ is by looping through the smallest end!

If both points $x, y$ belong to the same end $\mathcal{R}^{n_{i}} \backslash K$, then we have, for all $t>1$,

$$
p(t, x, y) \asymp \frac{1}{t^{n / 2}|x|^{n_{i}-2}|y|^{n_{i}-2}} e^{-\frac{|x|^{2}+|y|^{2}}{c t}}+\frac{1}{t^{n_{i} / 2}} e^{-\frac{d^{2}(x, y)}{c t}} .
$$

Let us emphasize that in all the estimates above, we have assumed $n_{i}>2$ for all $i$. If $n_{i} \leq 2$ for some $i$ then the heat kernel behaves differently as will be discussed in Section 4 - see Theorem 4 and Corollary 5.

The condition $n_{i}>2$ is equivalent to the non-parabolicity of $\mathcal{R}^{n_{i}}$. A manifold $M$ is called parabolic if the Brownian motion $X_{t}$ on $M$ is recurrent, and nonparabolic otherwise. The non-parabolicity is equivalent to the existence of a positive fundamental solution of the Laplace operator (see the survey [13] for detailed account of parabolicity).

In our main results - Theorems 1 and 4 below, we will distinguish the cases when all the ends $M_{i}$ are non-parabolic or not.

\section{The case when all the ends are non-parabolic}

For any manifold $M_{i}$, denote by $d_{i}$ the geodesic distance on $M_{i}$, by $B_{i}(x, r)$ the geodesic ball on $M_{i}$ of the radius $r$ centered at $x$, and by $V_{i}(x, r)$ the Riemannian volume of that ball. Also, let $p_{i}$ be the heat kernel of $M_{i}$. Note that the non-parabolicity of $M_{i}$ is equivalent to

$$
\int^{\infty} p_{i}(t, x, x) d t<\infty
$$

for some/all $x \in M_{i}$. If $M_{i}$ is geodesically complete and, for some $x \in M_{i}$,

$$
\int^{\infty} \frac{d t}{V_{i}(x, \sqrt{t})}=\infty
$$

then $M_{i}$ is parabolic - see [5], [10], [14], [20].

Let us introduce the following hypotheses, which in general may be true or not.

(i) The volume doubling condition: for some $C>0$ and for all $r>0$, $x \in M_{i}$,

$$
V_{i}(x, 2 r) \leq C V_{i}(x, r) .
$$

(ii) The on-diagonal upper bound of the heat kernel: for some $C>0$ and for all $t>0, x \in M_{i}$,

$$
p_{i}(t, x, x) \leq \frac{C}{V_{i}(x, \sqrt{t})} .
$$

(iii) The off-diagonal lower bound of the heat kernel : for some $C>0$ and for all $t>0, x, y \in M$, 


$$
p_{i}(t, x, y) \geq \frac{C^{-1}}{V_{i}(x, \sqrt{t})} \exp \left(-C \frac{d_{i}^{2}(x, y)}{t}\right)
$$

For example, all the hypotheses (i), (ii) and (iii) hold if $M_{i}$ has non-negative Ricci curvature - see [16]. Note that, under the hypotheses (i) and (ii), the condition (10) is not only sufficient but also necessary for the parabolicity of $M_{i}$. Let us also mention that (i) and (ii) imply an off-diagonal upper bound for the heat kernel matching (12) (see [12]) as well as an on-diagonal lower bound matching (11) (see [6]). The necessary and sufficient conditions for (i) and (ii) are given in terms of a relative Faber-Krahn inequality — see [12]. The hypotheses (i),(ii) and (iii) are equivalent to a parabolic Harnack inequality, which, in turn, is equivalent to the volume doubling (i) and a Poincaré inequality — see [17] and [11].

In order to state the heat kernel upper bound on the manifold $M$, let us introduce the following notation. Denote as above

$$
|x|=\sup _{z \in K} d(x, z)
$$

and define the new distances:

1. $d_{+}(x, y)$ is the infimum of lengths of smooth curves connecting $x$ and $y$ in $M$ and intersecting $K$;

2. $d_{\emptyset}(x, y)$ is the infimum of lengths of smooth curves connecting $x$ and $y$ in $M$ and not intersecting $K$.

Clearly, both $d_{+}(x, y)$ and $d_{\emptyset}(x, y)$ are larger than or equal to the geodesic distance $d(x, y)$, and one of them is equal to $d(x, y)$. If $x$ and $y$ are at different ends then $d_{\emptyset}(x, y)=\infty$.

For each $i \geq 1$, fix a reference point $o_{i} \in \partial K \cap M_{i}$, set

$$
\begin{aligned}
W_{i}(r) & =V_{i}\left(o_{i}, r\right), \\
W_{\min }(r) & =\min _{i} W_{i}(r),
\end{aligned}
$$

and define the function $W(x, r)$ by

$$
W(x, r)= \begin{cases}W_{i}(r), & x \in E_{i} \\ W_{\min }(r), & x \in K\end{cases}
$$

In words, $W_{i}(r)$ is the volume growth function for the manifold $M_{i}, W_{\min }(r)$ is the smallest volume growth, and $W(x, r)$ switches between $W_{i}$ and $W_{\min }$ depending on the location of $x$.

Finally, let us define the function $H(x, t)$, for all $t>0$ and $x \in M$, by

$$
\begin{array}{ll}
H(x, t)=\frac{|x|^{2}}{W_{i}(|x|)}+\left(\int_{|x|^{2}}^{t} \frac{d s}{W_{i}(\sqrt{s})}\right)_{+}, & \text {if } x \in E_{i}, \\
H(x, t)=1, & \text { if } x \in K .
\end{array}
$$


Theorem 1. Assume that all $M_{i}, i=1,2, \ldots, k$, are complete non-compact non-parabolic manifolds satisfying (i), (ii), and (iii). Then the heat kernel $p(t, x, y)$ on the connected sum $M=M_{1} \# M_{2} \# \cdots \# M_{k}$ admits the following estimate: for all $t>0$ and $x, y \in M$,

$$
\begin{aligned}
p(t, x, y) \asymp & \left(\frac{H(x, t)}{W(y, \sqrt{t})}+\frac{H(y, t)}{W(x, \sqrt{t})}+\frac{H(x, t) H(y, t)}{W_{\min }(\sqrt{t})}\right) e^{-\frac{d_{+}^{2}(x, y)}{c t}} \\
& +\frac{1}{\sqrt{V_{i}(x, \sqrt{t}) V_{i}(y, \sqrt{t})}} e^{-\frac{d_{\emptyset}^{2}(x, y)}{c t}}
\end{aligned}
$$

where the term in (19) refers to the case when both $x, y$ belong to the same end $E_{i}$ (otherwise $d=\infty$ ). If all $M_{i}$ satisfy only (i) and (ii) (but not necessarily (iii),) then the upper bound in (18)-(19) is still valid.

In particular, if $x \in E_{i}$ and $y \in E_{j}$ where $i \neq j$ then (18)-(19) yield

$$
p(t, x, y) \asymp\left(\frac{H(x, t)}{W_{j}(\sqrt{t})}+\frac{H(y, t)}{W_{i}(\sqrt{t})}+\frac{H(x, t) H(y, t)}{W_{\min }(\sqrt{t})}\right) e^{-\frac{d^{2}(x, y)}{c t}},
$$

because in this case $d_{\emptyset}(x, y)=\infty$ and $d_{+}(x, y)=d(x, y)$. If $x$ and $y$ lie at the same end, that is, $i=j$ then we obtain

$$
p(t, x, y) \asymp \frac{H(x, t) H(y, t)}{W_{\min }(\sqrt{t})} e^{-\frac{|x|^{2}+|y|^{2}}{c t}}+\frac{1}{V_{i}(x, \sqrt{t})} e^{-\frac{d^{2}(x, y)}{c t}} .
$$

Let us show examples of computation of the function $H$.

Example 1. Suppose that, for all $i$, the volume growth on $M_{i}$ is given by

$$
W_{i}(r) \asymp r^{n_{i}}, \quad \text { for } r>1,
$$

where all $n_{i}>2$. The latter implies, in particular, (9) whence $M_{i}$ is nonparabolic. Next, we have

$$
W_{\text {min }} \asymp r^{n} \text {, }
$$

where $n=\min _{i} n_{i}$. Assuming $x \in E_{i}$ and $|x|>1$, we compute $H(x, t)$ by

$$
H(x, t) \asymp|x|^{2-n_{i}}+\left(\int_{|x|^{2}}^{t} \frac{d s}{s^{n_{i} / 2}}\right)_{+} \asymp|x|^{2-n_{i}} .
$$

Hence, the estimates (20) and (21) become (7) and (8), respectively.

Example 2. Suppose now that, for all $i$, the volume growth function is as follows

$$
W_{i}(r) \asymp r^{2} \log ^{\alpha_{i}} r, \quad r>2,
$$

where $\alpha_{i}>1$ (which ensures the non-parabolicity of $M_{i}$ ). Then

$$
W_{\min } \asymp r^{2} \log ^{\alpha} r
$$


where

$$
\alpha=\min _{i} \alpha_{i}
$$

Assuming $x \in E_{i}$ and $|x|>2$, we compute $H(x, t)$ by

$$
\begin{gathered}
H(x, t)=\log ^{-\alpha_{i}}|x|+\left(\int_{|x|^{2}}^{t} \frac{d s}{s \log ^{\alpha_{i}} s}\right)_{+} \\
\quad \asymp \log ^{-\alpha_{i}}|x|+\left(\log ^{1-\alpha_{i}}|x|^{2}-\log ^{1-\alpha_{i}} t\right)_{+} .
\end{gathered}
$$

If $|x| \geq \sqrt{t}$ then $H(x, t) \asymp \log ^{-\alpha_{i}}|x|$. Assuming $y \in E_{j}$ and $|x| \asymp|y| \asymp \sqrt{t}$ (where $i \neq j$ ) we obtain from (20) the following medium time asymptotic

$$
p(t, x, y) \asymp \frac{1}{t \log ^{\alpha_{i}+\alpha_{j}} t} .
$$

If $|x|<<\sqrt{t}$ then $H(x, t) \asymp \log ^{1-\alpha_{1}}|x|$. Assuming that $|x|,|y|$ remain bounded while $t \rightarrow \infty$, we obtain the long time asymptotic

$$
p(t, x, y) \asymp \frac{1}{t \log ^{\alpha} t} .
$$

\section{The mixed case with both parabolic and non-parabolic ends}

We will need here the following additional hypothesis.

(iv) Connectedness of the annuli: for all $R$ large enough, the annulus $B_{i}\left(o_{i}, 4 R\right) \backslash B_{i}\left(o_{i}, R / 4\right)$ is connected ${ }^{1}$.

So far we have not required that the end $E_{i}$ is connected. Clearly, the connectedness of the annuli is even stronger assumption than the connectedness of $E_{i}$. Basically, the hypothesis (iv) prohibits the situation when $M_{i}$ consists of two "nearly" disjoint unbounded parts connected only by a rare sequence of small tubes.

It is easy to see that any two-dimensional convex surface in $\mathbb{R}^{3}$ satisfies all the hypotheses (i), (ii), (iii) and (iv), provided it is complete and unbounded. Also, all manifolds $\mathcal{R}^{n}$ introduced above satisfy these hypotheses.

We start with the following lemma.

Lemma 2. Suppose that all $M_{i}, i=1,2, \ldots, k$ are geodesically complete manifolds satisfying (i), (ii), (iii), and (iv). Assume that at least one of them is

\footnotetext{
${ }^{1}$ This hypothesis can be slightly relaxed as follows: for any two points $x, y$ both at distance $R$ from $o_{i}$, there is a continuous path connecting $x$ to $y$ and staying in the annulus $B_{i}\left(o_{i}, 4 R\right) \backslash$ $B_{i}\left(o_{i}, R / 4\right)$.
} 
non-parabolic. Then there exists a positive harmonic function $h(x)$ on $M=$ $M_{1} \# M_{2} \# \cdots \# M_{k}$ such that, for all $x \in M$,

$$
h(x) \asymp 1+\left(\int_{1}^{|x|^{2}} \frac{d s}{W(x, \sqrt{s})}\right)_{+}
$$

where $W$ is defined by (13)-(15).

In particular, $h(x) \asymp 1$ if $x$ stays in a non-parabolic end whereas $h(x) \rightarrow \infty$ if $x \rightarrow \infty$ within a parabolic end.

For any smooth positive function $h(x)$ on $M$, one may consider the weighted manifold $\widetilde{M}=\left(M, h^{2}\right)$ which means that, in addition to the Riemannian structure, $\widetilde{M}$ is endowed with the measure $\widetilde{\mu}$ defined by $d \widetilde{\mu}=h^{2} d \mu$ where $\mu$ is the Riemannian measure on $M$. There is a natural weighted Laplace operator on $\widetilde{M}$ defined by

$$
\widetilde{\Delta}=h^{-2} \operatorname{div}\left(h^{2} \nabla\right),
$$

which is formally self-adjoint with respect to $\widetilde{\mu}$. Denote by $\widetilde{p}$ the heat kernel of $\widetilde{\Delta}$. If $h(x)$ is, in addition, harmonic then it is easy to see that

$$
\widetilde{\Delta}=h^{-1} \circ \Delta \circ h
$$

which implies

$$
\widetilde{p}(t, x, y)=\frac{p(t, x, y)}{h(x) h(y)}
$$

Let us define the function $h_{i}(x)$ on $M_{i}$ as follows: $h_{i}(x)=h(x)$ if $x \in E_{i}$ and $h_{i}$ is extended to $K_{i}$ somehow, to remain smooth and positive. Clearly, we have, for all $x \in M_{i}$,

$$
h_{i}(x) \asymp 1+\left(\int_{1}^{|x|^{2}} \frac{d s}{W_{i}(\sqrt{s})}\right)_{+} .
$$

Consider the weighted manifold $\widetilde{M}_{i}=\left(M_{i}, h_{i}^{2}\right)$ and introduce similarly $\widetilde{\mu}_{i}, \widetilde{\Delta}_{i}$ and $\widetilde{p}_{i}$ (since $h_{i}$ is not necessarily harmonic on $M_{i}$, the kernel $\widetilde{p}_{i}$ does not necessarily satisfies the analogue of $(22))$. Denote $\widetilde{V}_{i}(x, r)=\widetilde{\mu}_{i}\left(B_{i}(x, r)\right)$.

Lemma 3. Suppose that all $M_{i}, i=1,2, \ldots, k$ are geodesically complete manifolds satisfying (i), (ii), (iii) and (iv). Assume that at least one of them is non-parabolic. Let $h$ and $h_{i}$ be the functions defined as above. Then each $\widetilde{M}_{i}$ is non-parabolic and satisfies the conditions (i), (ii) and (iii) (where one should naturally replace $V_{i}$ by $\widetilde{V}_{i}$ and $p_{i}$ by $\tilde{p}_{i}$ ). 
In fact, Theorem 1 remains true for weighted manifolds. Therefore, by Lemma 3 , one can apply Theorem 1 to the above setting, estimate $\widetilde{p}$, and then recover $p$ by (22). To state the result which we obtain in this way, we need the following notation. Let us set

$$
\widetilde{W}_{i}(r):=\widetilde{V}_{i}\left(o_{i}, r\right)
$$

and define $\widetilde{W}_{\min }(r), \widetilde{W}(x, r)$ and $\widetilde{H}(x, t)$ analogously to $(14),(15)$ and (16)-(17) where $W_{i}$ must be replaced everywhere by $\widetilde{W}_{i}$.

Theorem 4. Assume that all $M_{i}, i=1,2, \ldots, k$, are complete non-compact manifolds satisfying (i), (ii), (iii), (iv), and at least one of $M_{i}$ 's is nonparabolic. Then the heat kernel $p(t, x, y)$ on the connected sum $M=M_{1} \# M_{2} \# \ldots \# M_{k}$ admits the following estimate: for all $t>0$ and $x, y \in M$,

$$
\begin{array}{r}
p(t, x, y) \asymp h(x) h(y)\left(\frac{\widetilde{H}(x, t)}{\widetilde{W}(y, \sqrt{t})}+\frac{\widetilde{H}(y, t)}{\widetilde{W}(x, \sqrt{t})}+\frac{\widetilde{H}(x, t) \widetilde{H}(y, t)}{\widetilde{W}_{\min }(\sqrt{t})}\right) e^{-\frac{d_{+}^{2}(x, y)}{c t}} \\
+\frac{h(x) h(y)}{\sqrt{\widetilde{V}_{i}(x, \sqrt{t}) \widetilde{V}_{i}(y, \sqrt{t})}} e^{-\frac{d_{\emptyset}^{2}(x, y)}{c t}},
\end{array}
$$

where the last term refers to the case when both $x, y$ belong to the same end $E_{i}$ (otherwise $d=\infty$ ).

Denote

$$
\eta_{i}(r):=1+\left(\int_{1}^{r^{2}} \frac{d s}{W_{i}(\sqrt{s})}\right)_{+}
$$

Then (23) implies $h_{i}(x) \asymp \eta_{i}(|x|)$. It is possible to prove that

$$
\widetilde{W}_{i}(r) \asymp \eta_{i}^{2}(r) W_{i}(r)
$$

and, if $x \in E_{i}$,

$$
\widetilde{H}(x, t) \asymp \frac{|x|^{2}}{\eta_{i}^{2}(|x|) W_{i}(|x|)}+\frac{1}{\eta_{i}(|x|) \eta_{i}(\sqrt{t})}\left(\int_{|x|^{2}}^{t} \frac{d s}{W_{i}(\sqrt{s})}\right)_{+} .
$$

Let us show some examples how to apply Theorem 4 using (24)-(26).

Example 3. Let $M_{1}=\mathcal{R}^{1}:=\mathbb{R}_{+} \times \mathbb{S}^{N-1}, M_{2}=\mathcal{R}^{3}$ and consider the connected sum $M=\mathcal{R}^{1} \# \mathcal{R}^{3}$. We have, for $r>1$,

$$
W_{1}(r) \asymp r \quad \text { and } \quad W_{2}(r) \asymp r^{3} .
$$

By (24), we obtain, for $r>1$,

$$
\eta_{1}(r) \asymp r, \quad \text { and } \quad \eta_{2}(r) \asymp 1 .
$$


Then we compute $\widetilde{W}_{i}$ by $(25)$

$$
\widetilde{W}_{1}(r) \asymp r^{3}, \text { and } \widetilde{W}_{2}(r) \asymp r^{3},
$$

whence

$$
\widetilde{W}_{\min }(r)=\min \left(\widetilde{W}_{1}(r), \widetilde{W}_{2}(r)\right) \asymp r^{3} .
$$

Finally, let us compute $\widetilde{H}(x, t)$ and $\widetilde{H}(y, t)$ assuming $x \in E_{1}, y \in E_{2}$ and $|x|,|y|, t>1$. By (26), we obtain

$$
\widetilde{H}(x, t) \asymp|x|^{-1}, \quad \text { and } \quad \widetilde{H}(y, t) \asymp|y|^{-1} .
$$

Hence, Theorem 4 yields, for the chosen $x, y$ and $t$,

$$
p(t, x, y) \asymp \frac{1}{t^{3 / 2}}\left(1+\frac{|x|}{|y|}\right) e^{-\frac{d^{2}(x, y)}{c t}} .
$$

We see that the contributions of both ends $\mathcal{R}^{1}$ and $\mathcal{R}^{3}$ to the long time behavior of the heat kernel on $M$ are of the same order $t^{-3 / 2}$. This may seem surprising, in view of the heat estimate (4) for the manifold $\mathcal{R}^{n} \# \mathcal{R}^{m}$ with $n, m>2$, which contains both terms $t^{-n / 2}$ and $t^{-m / 2}$. The explanation is that what counts for the manifold $\mathcal{R}^{1} \# \mathcal{R}^{3}$ is the heat kernel long time behavior on $\widetilde{\mathcal{R}}^{1}$ rather than that on $\mathcal{R}^{1}$. However, $\widetilde{\mathcal{R}}^{1}$ has the volume growth $\widetilde{W}_{1}(r) \asymp r^{3}$ and, therefore, the heat kernel of the order $t^{-3 / 2}$. This effect was first observed by E.B. Davies [9] in a model situation of a one-dimensional complex.

Example 4. Let us take $M_{1}=\mathcal{R}^{2}$ and $M_{2}=\mathcal{R}^{3}$. We compute as in the previous example, for $r>2$ and $|x|>2$,

$$
\begin{gathered}
W_{1}(r) \asymp r^{2}, \quad \text { and } \quad W_{2}(r) \asymp r^{3}, \\
h_{1}(x) \asymp \log |x|, \quad \text { and } \quad h_{2}(x) \asymp 1, \\
\widetilde{W}_{1}(r) \asymp r^{2} \log ^{2} r, \quad \text { and } \quad \widetilde{W}_{2}(r) \asymp r^{3} .
\end{gathered}
$$

If $x \in E_{1}$ and $y \in E_{2}$ then, for $t>2$, we have $\widetilde{H}(y, t) \asymp|y|^{-1}$ whereas

$$
\widetilde{H}(x, t) \asymp \frac{1}{\log ^{2}|x|}+\left(\frac{1}{2 \log |x|}-\frac{1}{\log t}\right)_{+} .
$$

Hence, for the chosen $x, y, t$, we obtain

$$
p(t, x, y) \asymp\left(\frac{\log |x|}{|y| t \log ^{2} t}+\frac{1}{t^{3 / 2}}\left[\frac{1}{\log |x|}+\left(\frac{1}{2}-\frac{\log |x|}{\log t}\right)_{+}\right]\right) e^{-\frac{d^{2}(x, y)}{c t}}
$$

In particular, the long time asymptotic is as follows

$$
p(t, x, y) \asymp \frac{1}{t \log ^{2} t},
$$


whereas the medium time asymptotic (when $|x| \asymp|y| \asymp \sqrt{t}$ ) is given by

$$
p(t, x, y) \asymp \frac{1}{t^{3 / 2} \log t} .
$$

In certain situations, the formulas (24), (25) and (26) can be further simplified. Namely, let us assume that, for $r$ large enough,

$$
\int_{1}^{r^{2}} \frac{d s}{W_{i}(\sqrt{s})} \asymp \frac{r^{2}}{W_{i}(r)} .
$$

Then we have the following relations, for $r$ large enough,

$$
\begin{aligned}
\eta_{i}(r) & \asymp \frac{r^{2}}{W_{i}(r)}, \\
\widetilde{W}_{i}(r) & \asymp \frac{r^{4}}{W_{i}(r)}, \\
\widetilde{H}(x, t) & \asymp|x|^{-2} W_{i}(|x|),
\end{aligned}
$$

where in the last line $x \in E_{i}$ and $|x|, t$ are large enough. For example, (28) holds if $W_{i}(r) \asymp r^{\alpha}$ with $0<\alpha<2$. For this $W_{i}$, we obtain

$$
\begin{aligned}
\eta_{i}(r) & \asymp r^{2-\alpha}, \\
\widetilde{W}_{i}(r) & \asymp r^{4-\alpha}=r^{\alpha^{*}}, \\
\widetilde{H}(x, t) & \asymp|x|^{\alpha-2}=|x|^{2-\alpha^{*}},
\end{aligned}
$$

where

$$
\alpha^{*}:=4-\alpha .
$$

We see that $\widetilde{W}_{i}(r)$ and $\widetilde{H}(x, t)$ look like the corresponding functions on a nonparabolic manifold with volume growth $r^{\alpha^{*}}$. Hence, to some extent, the parabolic manifold with volume growth $r^{\alpha}$ can be regarded as dual to the non-parabolic manifold with volume growth $r^{\alpha^{*}}$.

Corollary 5. Under the hypotheses of Theorem 4, assume in addition that each manifold $M_{i}$ has volume growth of the order $r^{n_{i}}$ where $n_{i} \neq 2$. Denote

$$
n_{i}^{*}:= \begin{cases}4-n_{i}, & n_{i}<2, \\ n_{i}, & n_{i}>2,\end{cases}
$$

and

$$
n:=\min _{1 \leq i \leq k} n_{i}^{*} .
$$


If $x \in E_{i}, y \in E_{j}, i \neq j$, and $|x|,|y|, t$ are large enough then

$$
\begin{array}{r}
p(t, x, y) \asymp\left(\frac{1}{t^{n_{j}^{*} / 2}|x|^{n_{i}^{*}-2}}+\frac{1}{t^{n_{i}^{*} / 2}|y|^{n_{j}^{*}-2}}+\frac{1}{t^{n / 2}|x|^{n_{i}^{*}-2}|y|^{n_{j}^{*}-2}}\right) \\
|x|^{\left(2-n_{i}\right)_{+}}|y|^{\left(2-n_{j}\right)_{+}} e^{-\frac{d^{2}(x, y)}{c t}} .
\end{array}
$$

In particular, (29) contains the estimate (7) for the manifold $\mathcal{R}^{n_{1}} \# \mathcal{R}^{n_{2}} \# \cdots \# \mathcal{R}^{n_{k}}$ when all $n_{i}$ are larger than 2 . The estimate $(27)$ for the manifold $\mathcal{R}^{1} \# \mathcal{R}^{3}$ is also a straightforward consequence of (29).

The long time asymptotic in (29) is determined by the term $t^{-n / 2}$. This was noticed by Davies [9] for an one-dimensional complex, modelling manifolds with ends. If all $n_{i}>2$ then $n / 2$ can be interpreted as the exponent of the largest heat kernel of $M_{i}$ 's. However, in general this is not true. It turns out that $t^{-n_{i}^{*} / 2}$ is the rate of decay of the Dirichlet heat kernel of $E_{i}$, that is, the heat kernel on $E_{i}$ with the vanishing boundary condition on $\partial E_{i}$. Therefore, the term $t^{-n / 2}$ is determined in general by the largest Dirichlet heat kernel on $E_{i}$ 's. In fact, precise estimates of the Dirichlet heat kernel on $E_{i}$ are crucial tools for the proof of the results described above.

Assume $n_{i}<2, n_{j}>2$ and consider the medium time asymptotic regime when $|x| \asymp \sqrt{t}$ and $t \rightarrow \infty$, in which case (29) implies

$$
p(t, x, y) \asymp \frac{1}{t^{n_{j}^{*} / 2}}+\frac{1}{t|y|^{n_{j}^{*}-2}} .
$$

If also $|y| \asymp \sqrt{t}$ then the first term in (30) is leading. Hence, the medium time asymptotic is determined only by the larger end, in contrast to the case when both ends are non-parabolic (c.f., (5)).

However, the most interesting paradoxical effect occurs if we let $|y| \asymp 1$ in (30). In this case,

$$
p(t, x, y) \asymp t^{-1}
$$

regardless of the exponents $n_{i}, n_{j}$ ! Therefore, if $x$ moves away at the rate $\sqrt{t}$ and $y$ stays near the central part, then the heat kernel $p(t, x, y)$ given by $(31)$ is larger than in the long time regime when both $x$ and $y$ stay near the central part.

The explanation is that if $x$ and $y$ are close to the central part and $t$ is large then the process $X_{t}$ started at $x$ tends to escape to infinity within the larger end so that its chances to loop back to $y$ are relatively small. On the contrary, if $X_{t}$ starts at the point $x$ located at the smaller end at the distance $\sqrt{t}$ from the central part, then it cannot escape to infinity within this end because of its parabolicity. Hence, it moves towards the central part and hits $y$ in time $t$ with a higher probability.

The hypothesis of Theorem 4 and Corollary 5 that at least one end $M_{i}$ is nonparabolic is equivalent to the non-parabolicity of $M-$ see [10]. If all $M_{i}$ 's are 
parabolic and, thus, $M$ is also parabolic, then the estimate (29) cannot be true. Indeed, the long time decay $t^{-n / 2}$ given by (29) implies (9), which contradicts the parabolicity of $M$.

The case when all $M_{i}$ are parabolic will be addressed elsewhere.

\section{Acknowledgment}

Both authors were supported by the travel grants of the EU Stochastic Analysis Network.

\section{References}

[1] J-Ph. Anker, L. Ji Heat kernel and Green function estimates on non-compact symmetric spaces, preprint, 1998

[2] I. Benjamini, I. Chavel, and E.A. Feldman, Heat kernel lower bounds on Riemannian manifolds using the old ideas of Nash, Proc. London Math. Soc. (3) 72 (1996) 215-240.

[3] J. Cheeger, M. Gromov, and M. Taylor, Finite propagation speed, kernel estimates for functions of the Laplace operator, and the geometry of complete Riemannian manifolds, J. Differential Geom. 17 (1982) 15-53.

[4] J. Cheeger and S.-T. Yau, A lower bound for the heat kernel, Comm. Pure Appl. Math. 34 (1981) 465-480.

[5] S.Y. Cheng and S.-T. Yau, Differential equations on Riemannian manifolds and their geometric applications, Comm. Pure Appl. Math. 28 (1975) 333-354.

[6] T. Coulhon and A. Grigor'yan, On-diagonal lower bounds for heat kernels on noncompact manifolds and Markov chains, Duke Math. J. 89 (1997) 133-199.

[7] E.B. Davies, Explicit constants for Gaussian upper bounds on heat kernels, Amer. J. Math. 109 (1987) 319-334.

[8] _ Gaussian upper bounds for the heat kernel of some second-order operators on Riemannian manifolds, J. Funct. Anal. 80 (1988) 16-32.

[9] , Non-Gaussian aspects of heat kernel behaviour, J. London Math. Soc. (2) 55 (1997) 105-125.

[10] A. Grigor'yan, On the existence of positive fundamental solution of the Laplace equation on Riemannian manifolds, (Russian) Mat. Sb. (N.S.) 128 (1985) 354-363. Engl. transl. in Math. USSR Sb. 56 (1987) 349-358.

[11] A. Grigor'yan, The heat equation on non-compact Riemannian manifolds,(Russian) Matem. Sbornik 182 (1991) 55-87. Engl. transl. in Math. USSR Sb. 72 (1992) 47-77.

[12] _ Heat kernel upper bounds on a complete non-compact manifold, Rev. Mat. Iberoamericana 10 (1994) 395-452.

[13] _ Analytic and geometric background of recurrence and non-explosion of the Brownian motion on Riemannian manifolds, Bull. Amer. Math. Soc. (N.S.) 36 (1999) 135249.

[14] L. Karp, Subharmonic functions, harmonic mappings and isometric immersions, Seminar on Differential Geometry, (S.T. Yau, ed.) Ann. Math. Stud. 102, Princeton University Press, Princeton, N.J., 1982.

[15] Yu.T. Kuz'menko and S.A. Molchanov, Counterexamples to Liouville-type theorems, (Russian) Vestnik Moskov. Univ. Ser. I Mat. Mekh. (1979) 39-43. Engl. transl. in Moscow Univ. Math. Bull. 34 (1979) 35-39.

[16] P. Li and S.-T. Yau, On the parabolic kernel of the Schrödinger operator, Acta Math. 156 (1986) no.3-4, 153-201.

[17] L. Saloff-Coste, A note on Poincaré, Sobolev, and Harnack inequalities, Duke Math J., Internat. Math. Res. Notices 2 (1992) 27-38. 
[18] _ Uniformly elliptic operators on Riemannian manifolds, J. Diff. Geom. 36 (1992) $417-450$.

[19] Yu.A. Semenov, Heat kernel bounds. $L^{1}$-iterative techniques. The Nash algorithm, preprint, 1999.

[20] N.Th. Varopoulos, Potential theory and diffusion of Riemannian manifolds, Conference on Harmonic Analysis in honor of Antoni Zygmund. Vol I, II., pp. 821-837, Wadsworth Math. Ser., Wadsworth, Belmont, Calif., 1983.

Department of Mathematics, 180 Queen's Gate, Huxley Building, Imperial ColLEGE, London SW7 2BZ, United Kingdom

E-mail address: a.grigoryan@ic.ac.uk

CNRS, Toulouse, France,

Department of Mathematics, Malott Hall, Cornell University, Ithaca, Ny 148534201

E-mail address: lsc@math.cornell.edu 